\title{
Dissociable neural representations of wrist motor coordinate frames in human motor cortices
}

Natsue Yoshimura ${ }^{1,2}$, Koji Jimura ${ }^{1,2,3}$, Charles Sayo DaSalla ${ }^{3}$, Duk Shin ${ }^{1,3}$, Hiroyuki

5 Kambara $^{1,3}$, Takashi Hanakawa ${ }^{2,4,5}$, and Yasuharu Koike $e^{1,3,6}$

1 Precision and Intelligence Laboratory, Tokyo Institute of Technology, Yokohama 226-8503, Japan

2 Department of Functional Brain Research, National Institute of Neuroscience,

10 National Center of Neurology and Psychiatry, Tokyo 187-8502, Japan

${ }^{3}$ CREST, Japan Science and Technology Agency, Tokyo 102-0076, Japan

${ }^{4}$ Department of Advanced Neuroimaging, Integrative Brain Imaging Center, National Center of Neurology and Psychiatry, Tokyo 187-8551, Japan

${ }^{5}$ PRESTO, Japan Science and Technology Agency, Tokyo 102-0076, Japan

6 Solution Science Research Laboratory, Tokyo Institute of Technology, Yokohama 226-8503, Japan

Corresponding author: Natsue Yoshimura

E-mail address: yoshimura@cns.pi.titech.ac.jp

20 Postal address: 4259-J3-10, Nagatsuta-cho, Midori-ku, Yokohama 226-8503, Japan.

Phone: +81-45-924-5054

Fax: $\quad+81-45-924-5085$ 


\section{Abstract}

There is a growing interest in how the brain transforms body part positioning in the extrinsic environment into an intrinsic coordinate frame during motor control. To explore the human brain areas representing intrinsic and extrinsic coordinate frames,

5 this fMRI study examined neural representation of motor cortices while human participants performed isometric wrist flexions and extensions in different forearm postures, thereby applying the same wrist actions (representing the intrinsic coordinate frame) to different movement directions (representing the extrinsic coordinate frame). Using sparse logistic regression, critical voxels involving pattern information that specifically discriminates wrist action (flexion vs. extension) and movement direction (upward vs. downward) were identified within the primary motor and premotor cortices. Analyses of classifier weights further identified contributions of the primary motor cortex to the intrinsic coordinate frame and the ventral and dorsal premotor cortex and supplementary motor area proper to the extrinsic coordinate frame. These results are consistent with existing findings using non-human primates and demonstrate the distributed representations of independent coordinate frames in the human brain.

Keywords: motor areas, motor coordinate frames, functional magnetic resonance imaging, multivariate pattern analysis, voluntary movement 


\section{Introduction}

The brain allows for skillful manipulation of the body to interact with the external environment. This sophisticated and flexible operation involves transformations between coordinate frames of the internal body and external environment, possibly

5 computed in distributed brain regions. The intrinsic coordinate frame is body- and/or muscle-centered, whereas the extrinsic coordinate frame refers to points outside the body. However, it is still unclear how these two coordinate frames are represented in the brain.

Extensive studies using monkeys have shown that the primary motor cortex (M1)

10 and the premotor cortex (PM) are important in coding coordinate frames. M1 has been found to represent not only the intrinsic coordinate frame, through parameters such as muscle tension (Cheney et al., 1985; Donoghue et al., 1992; Evarts, 1968; Kakei et al., 1999) and joint angle (Scott and Kalaska, 1995), but also the extrinsic coordinate frame in the form of movement direction (Georgopoulos et al., 1986; Kakei et al., 15 1999). The ventral and dorsal regions of PM (PMv and PMd) have been found to be associated with the extrinsic coordinate frame. PMv codes the direction of action (Kakei et al., 2001), whereas PMd codes motor preparation (Kurata, 1993) and the 
relative position of targets for reaching (Pesaran et al., 2006). The supplementary motor area (SMA) is also included in the medial portion of PM. To our knowledge, no previous studies have examined the neural representation of distinct coordinate frames in SMA. However, the roles of the anatomically subdivided areas, pre-SMA and SMA 5 proper, seem to be relatively different. Evidence for these areas suggests that pre-SMA activates in relation to visual cues (Matsuzaka et al., 1992) or new motor plans (Shima et al., 1991), whereas SMA proper activates in relation to somatosensory stimuli (Matsuzaka et al., 1992).

These observations are further extended by human functional magnetic resonance imaging (fMRI), transcranial magnetic stimulation (TMS), and positron emission tomography (PET) studies. M1 has been found to be involved in both the intrinsic and extrinsic coordinate frames (Alaerts et al., 2009; Eisenberg et al., 2010; Toxopeus et al., 2011). A TMS study showed that movement observation lead to highly muscle-specific resonating activity in M1, indicating representation of the intrinsic coordinate (Alaerts et al., 2009). Conversely, fMRI studies using a "center-out task" showed that M1 encodes direction of movement, i.e., the extrinsic coordinate frame (Eisenberg et al., 2010; Toxopeus et al., 2011). TMS studies have shown the involvement of PMd in motor preparation (Davare et al., 2006) and action prediction (Duque et al., 2012; 
Stadler et al., 2012), and TMS and PET studies have indicated a role for PMv in motor imagery, motor preparation, and grip force prediction (Dafotakis et al., 2008; Davare et al., 2009; Stephan et al., 1995). Although evidence of non-primary motor areas in the human cortex have not shown complete homology with those in monkeys, findings

5 from both species suggest that PMd and PMv may be involved primarily in the extrinsic coordinate frame, because motor preparation and action prediction likely require position information of the body parts in external space.

Studies using PET or regional cerebral blood flow measurement found activation in pre-SMA for externally cued or unfamiliar movement tasks and in SMA proper for self-paced or familiar tasks (Deiber et al., 1991; Grafton et al., 1992; Jenkins et al., 1994; Jenkins et al., 2000; Playford et al., 1992). These findings may suggest that SMA proper is more strongly associated with direct motor control than pre-SMA. Though electrophysiological studies have been performed to examine the neural representations of motor coordinate frames in monkeys (Kakei et al., 1999, 2001), 15 performing similar studies in humans poses practical challenges. fMRI offers an attractive solution because it is a non-invasive approach and can be used to examine M1 and PM regions in their entirety, a capability impossible for electrophysiological methods focusing on microscopic areas. In order to extract such mutually transformed 
coordinate representations using fMRI data, increased analysis power is likely needed to detect experimental manipulations. Multivariate pattern analysis (MVPA) is a machine learning technique that can be applied to fMRI data, and its utility has become increasingly apparent due to its high sensitivity to experimental manipulation and areal dissociations (Kriegeskorte, 2011; Mourao-Miranda et al., 2005). Notably, it detects fine-grained pattern differences not found in regional-average activation by conventional univariate analyses and discovers novel neural mechanisms (Kamitani and Tong, 2005; Kriegeskorte, 2011; Miyawaki et al., 2008; Mur et al., 2009). MVPA may, thus, be suitable to dissociate neural representations of the intrinsic and extrinsic coordinate frames.

In this fMRI study, we directly addressed these issues by applying a novel approach to the representation of wrist action (intrinsic) and movement direction (extrinsic). During fMRI acquisition, healthy human participants performed isometric wrist flexion and extension tasks (Fig. 1) in different forearm postures, allowing independent manipulation of the intrinsic and extrinsic coordinate frames. In order to identify brain areas associated with the respective coordinate frames, two binary classifiers were trained based on sparse logistic regression (SLR) (Yamashita et al., 2008), a type of MVPA, for discriminating voxel pattern information for flexion vs. 
extension (FvE) movements and upward vs. downward (UvD) movements. Taking

advantage of SLR, which can train high-dimensional classifiers without prior

dimension reduction, binary classifier weight values for M1 and PM regions were

examined to identify brain areas associated with the intrinsic and extrinsic coordinate

5 frames.

\section{Materials and Methods}

\subsection{Participants}

Ten right-handed healthy human participants ( 2 females and 8 males), between 21 and

47 years of age (mean: 34.1, standard deviation (SD): 10.7), participated in this study.

10 Written informed consent was obtained from all participants prior to the experiment.

The experimental protocol was approved by the ethics committee of the National Center of Neurology and Psychiatry.

\section{2 fMRI experimental tasks}

Participants performed isometric flexions and extensions of the right wrist while their

right forearms were held in one of three different wrist postures: pronated (Pro; palm downward), supinated (Sup; palm upward), and midway between the two (Mid; palm 
leftward) (Fig. 1A). The right wrist was immobilized with two plastic splints and Velcro tape to prevent bending of the wrist joint. Wrist posture was fixed by locking the splints to a platform attached to the MRI scanner bed. For all three postures, participants performed three isometric tasks: a flexion task (Flex), an extension task (Ext), and a 5 no-force task (Still) serving as the control condition.

Before starting the experiment, participants practiced all tasks in all postures while paying particular attention to maintaining consistent muscle activity across conditions by monitoring real-time muscle activity levels calculated from surface electromyography (EMG) signals (Koike and Kawato, 1995; Yoshimura et al., 2012).

10 Exertion force level of flexor and extensor muscles was set to less than $30 \%$ of maximum voluntary contraction level. EMG signals were also recorded during fMRI scanning, and mean muscle activity levels were compared across conditions to determine the consistency of muscle activity levels after the experiment.

Each participant underwent three functional runs in the MRI scanner, one for each 15 of the three wrist postures (Pro, Sup, and Mid). Though it would be ideal if the posture was altered to mix the three conditions in single runs to ensure independence of training and test sets (Pereira et al., 2009), it was impossible to mix all conditions in one functional run due to fixation of the right arm. We instead tried to minimize the effect of 
this experimental limitation through data processing (described in 2.6 Sparse logistic regression) and statistical analysis of classifier performance using permutation tests (described in 2.7 Binary classification analysis from SLR). The order of the postures was counterbalanced across participants. One functional run consisted of eighteen $18 \mathrm{~s}$ task blocks (Flex, Ext, and Still, 6 times each; Fig. 1B), with a 3 s rest period between the task blocks. The order of the task blocks was pseudo-randomized such that all three types of task blocks appeared in every three consecutive blocks. Within a task block, participants repeated a task (i.e., force exertion) six times, with each exertion lasting $2 \mathrm{~s}$ interspersed with $1 \mathrm{~s}$ rest periods. projected on a screen and viewed through a mirror attached to the MRI head coil. Importantly, one arrow direction cued multiple tasks depending on wrist posture, allowing the dissociation of wrist actions and movement directions by classifier training. For example, an up arrow indicated Ext in Pro (ExtUp), whereas it indicated Flex in Sup (FlexUp) (Fig. 1A). In the case of the Still condition, a bar was presented instead of an arrow (Fig. 1B). Because participants lay in a supine position, upward movement corresponded to exerting force towards the head and downward movement to force towards the feet. 


\subsection{Data acquisition}

A 3 T Magnetom Trio MRI scanner equipped with an 8-channel array coil (Siemens,

Erlangen, Germany) was used for the fMRI experiment. Functional data were acquired with a $\mathrm{T} 2 *$-weighted gradient-echo, echo planar imaging sequence using the following 5 parameters: repetition time $(\mathrm{TR})=3 \mathrm{~s}$; echo time $(\mathrm{TE})=30 \mathrm{~ms}$; flip angle $(\mathrm{FA})=90^{\circ}$; field of view $(\mathrm{FOV})=192 \times 192 \mathrm{~mm}$; matrix size $=64 \times 64 ; 36$ slices; slice thickness $=$ $3 \mathrm{~mm} ; 140$ volumes. Then, a 3D anatomical image was acquired using an MP-RAGE T1-weighted sequence $\left(\mathrm{TR}=2 \mathrm{~s} ; \mathrm{TE}=4.38 \mathrm{~ms} ; \mathrm{FA}=8^{\circ} ; \mathrm{FOV}=192 \times 192 \mathrm{~mm} ;\right.$ matrix size $=192 \times 192 ; 160$ slices $;$ slice thickness $=1 \mathrm{~mm})$.

Gilching, Germany) to ensure that the participants performed all tasks properly with consistent force levels across conditions (Fig. 2). Two pairs of $2 \mathrm{~cm}$-spaced $\mathrm{Ag} / \mathrm{AgCl}$ electrodes were placed over the major muscles for wrist flexion and extension, the right flexor carpi radialis and right extensor carpi radialis brevis, respectively. Signals were acquired at a sampling rate of $5000 \mathrm{~Hz}$ and band-pass filtered from $0.016-1000 \mathrm{~Hz}$ using BrainVision Recorder software (Brain Products). The amplifier was set to a resolution of $0.5 \mu \mathrm{V}$ and a range of $\pm 16 \mathrm{mV}$. 


\subsection{Data preprocessing}

fMRI data were preprocessed using SPM8 (Wellcome Department of Cognitive Neurology, UK; available at http://www.fil.ion.ucl.ac.uk/spm), running on MATLAB R2011b (The MathWorks, Inc., Natick, MA). All functional images were first realigned 5 to the mean image of each functional run, and the aligned echo planar images were all co-registered to the participants' individual T1-weighted anatomical images, which were co-registered to the mean image of the first run. This co-registration allowed for alignment of all functional volumes and also inverse-normalization of a region of interest (ROI) mask (described in 2.5 Region of interest mask) defined in standard Montreal Neurological Institute (MNI) space. No spatial smoothing was applied to the functional images.

Inverse-normalization transformation matrices from an MNI template to individual participants' native brain space were estimated based on T1-weighted structural images, allowing conversion of the ROI mask anatomically defined in a standard MNI template

15 to the participants' native space.

\subsection{Region of interest mask}

The present study specifically targeted PM (Georgopoulos et al., 1986; Kakei et al., 
2001; Stadler et al., 2012) and M1 (Kakei et al., 1999; Toxopeus et al., 2011) to examine final output of motor control. The ROI was defined based on the Wake Forest University PickAtlas Version 3.0 (Wake Forest University, Winston-Salem, NC; available at http://fmri.wfubmc.edu/software/PickAtlas; (Lancaster et al., 1997;

5 Lancaster et al., 2000; Maldjian et al., 2004; Maldjian et al., 2003). We used a merged region of Brodmann areas 4 and 6, which included both M1 and PM. The inclusion of the two cortices in the ROI was also ensured by additional 3D dilation by two voxels to encompass the full range of the area and compensate for inhomogeneity between the participants' inverse-normalization transformation matrices, used in the following process. This original ROI mask was then inversely normalized to the native space of the individual participants. The number of voxels included in the ROI mask thus differed between participants, but ranged from 3,756 to 6,705 (mean: 4,632; SD: 879).

\subsection{Sparse logistic regression}

This study utilized SLR (Yamashita et al., 2008) to find voxel pattern information 
significance of each voxel within the entire M1 and PM to the classifications can be assessed using a single and common index. In order to ensure the specificity of the classifiers to each coordinate frame in a more general context, classification accuracies were inspected using an independent dataset that was not used for classifier training. SLR was applied within the ROI in participants' individual native space. Using preprocessed data described in the previous section, the time series functional data were extracted at six time points per block, providing 36 scans for each task. To minimize dependency among blocks, we did not use high-pass filtering but, rather, a subtraction method. Specifically, at each permutation of the three task blocks, mean signal intensity was calculated from the 6 scans of the Still task block and subtracted from the mean signal intensities of the Flex and Ext blocks, calculated from their respective 6 scans.

To perform coordinate-frame specific classifications, data of two different postures (Pro and Sup) were merged according to classifier type (Fig. 3). For example, the FlexUp task was treated as identical to FlexDown in the Flex vs. Ext (FvE) classification, and identical to ExtUp in the Up vs. Down (UvD) classification. Therefore, in FvE classification, FlexDown and FlexUp were classified versus ExtDown and ExtUp. Importantly, data from the Mid posture condition were not used for training the two classifiers but were used for training another independent classifier 
for FlexLeft vs. ExtRight (FLvER) classification. Furthermore, the Mid data were later used to test if the FvE and UvD classifiers truly comprised information exclusive to the intrinsic and extrinsic coordinate frames. The classifiers were trained based on L1-norm based SLR with Laplace approximation using SLR Toolbox version 1.2.1 alpha

5 (Advanced Telecommunications Research Institute International, Japan; available at http://www.cns.atr.jp/ oyamashi/SLR_WEB.html) (Yamashita et al., 2008). Weight matrices estimation was performed using six-fold leave-one-block-out cross validation. More specifically, each task had six data blocks, and five blocks from each task (20 in total) were used to train a classifier. The one remaining block from each task (four in total) was used to evaluate the performance of the trained classifier. This was repeated six-fold, with each fold using a unique partition of training and testing blocks.

\subsection{Binary classification analysis from SLR}

To examine classification performance of estimated classifiers for the intrinsic and the extrinsic coordinate frames, classification accuracies were calculated for FvE and UvD classifications (Fig. 4).

Mean classification accuracies for UvD (Fig. 4A, left), FvE (Fig. 4A, right) and FLvER (Fig. 4B, left) classifications were first calculated using each participant's mean 
accuracy from six-fold cross validation. Then the weight values of the UvD and FvE classifiers were respectively averaged and applied to the data from the Mid posture condition (Fig. 4B, center and right). To minimize dependency effects caused by the limitations to performance validation, we did not use parametric methods for statistical analyses but, instead, used non-parametric permutation tests (Nichols and Holmes, 2002; Stelzer et al., 2013). To compare accuracy of each participant with chance level, six-fold leave-one-block-out cross-validation was repeated 100 times by randomly permuting the class labels. The labeling with the highest overall accuracy would then have a $p$-value of $1 / 100=0.01$. For group comparison with chance level or with another classification, mean difference was evaluated 2,000 times using pseudo-randomized labels. The labeling with the highest overall difference would then have a $p$-value of $1 / 2000=0.0005$.

\subsection{Measuring contribution through group and ROI analyses of weight matrices}

We examined weight values in M1, PMd, PMv, pre-SMA, and SMA proper for FvE and

15 UvD classifiers (Fig. 5). Focal areas were defined, without bias to experimental conditions, as 6-mm spheres centered at local maxima in an SPM group-level univariate activation map for the contrast between all tasks and baseline. The identification of the 
local maxima was statistically validated with small volume correction within each of the brain areas. The small volumes were defined based on the Human Motor Area Template (HMAT) (Mayka et al., 2006), created based on a meta-analysis of more than 100 articles describing motor-related areas. In the template, we used the areas labeled M1, 5 PMd, PMv, pre-SMA, and SMA proper in the left hemisphere.

Contribution of sphere focal areas to the intrinsic and the extrinsic coordinate frames was examined by comparing their mean weight values for FvE and UvD classifiers. More specifically, mean of the absolute weight matrices of individual participants' FvE and UvD classifiers were normalized by the maximum weight value and converted to Analyze-formatted image files (Mayo Foundation, Rochester, MN) using MATLAB. After the image files were spatially normalized into MNI standard space, mean weight values across participants were calculated using SPM8 image calculator. Mean weight values of voxels within the focal areas were then compared for FvE and UvD classifiers (Fig. 5B). This analysis was performed to show whether the 5 focal areas (HMAT-ROIs M1, PMd, PMv, pre-SMA, and SMA proper) represent the intrinsic or extrinsic coordinate frames. Statistical significance between FvE and UvD classifications in each focal area was assessed by randomly permuting the labels and calculating mean difference between the two classifications 2,000 times. 


\section{Results}

\subsection{Consistency of muscle activity levels across conditions}

No significant differences were observed when comparing flexor and extensor muscle activities for flexion and extension tasks, respectively (Fig. 2) (Flexor: FlexUp vs.

$5 \quad$ FlexDown, $p=0.56$; FlexDown vs. FlexLeft, $p=0.39$; FlexUp vs. FlexLeft, $p=0.14$; Extensor: ExtUp vs. ExtDown, $p=0.81$; ExtDown vs. ExtRight, $p=0.51$; ExtUp vs. ExtRight, $p=0.66$, by permutation test). These results suggest that differences in brain activity during task execution were attributed to differences in wrist action type or movement direction, and not muscle activity level.

\subsection{Binary classification accuracies from SLR}

Figure 4A shows mean classification accuracies for two binary classifiers (FvE and UvD) created using SLR across participants. Both accuracies were significantly higher than chance level ( $p=0.0005$ for both), without significant difference between them ( $p$ $=0.80)$. These results suggest that both of the datasets include necessary pattern

15 information for each binary classification and have the ability to dissociate wrist action $(\mathrm{FvE})$ and movement direction $(\mathrm{UvD})$, respectively.

The binary classifiers for FvE and UvD were then applied to an independent data set, 
the Mid condition (Fig. 1A, right) to test whether the classifiers could discriminate voxel pattern information specific to wrist action or movement direction. Classification accuracy for FvE was significantly higher than chance level $(66.9 \%, p=0.0005$; Fig 4B right), whereas UvD showed only chance level accuracy $(46.9 \%, p=0.50$; Fig 4B

5 middle). The difference in accuracy between the two classifiers was also significant (FvE vs. UvD, $p=0.006$, Bonferroni corrected), and the UvD classifier showed significantly lower accuracy than that of the FLvER classifier (FLvER vs. UvD: $p=$ 0.002, FLvER vs. FvE: $p=0.08$, Bonferroni corrected). These results indicate that the Mid data set included the necessary pattern information for FLvER classification, such that classification accuracy was significantly higher than chance level $(p=0.0005)$ without significant differences with FvE and UvD classifier accuracies on Pro and Sup data (Fig. 4A) (FLvER vs. UvD: $p=0.12$, FLvER vs. FvE: $p=0.07$ ). They also suggest that the FvE classifier, but not the UvD classifier, holds voxel pattern information for dissociating FlexLeft and ExtRight. In other words, the FvE classifier seems to hold information specific to the intrinsic coordinate frame, whereas the UvD classifier does not. The UvD classifier does, however, seem to hold information for the extrinsic coordinate frame for Up and Down.

Thus far, we had trained cross-posture classifiers using two datasets with different 
postures (Pro and Sup), aiming to extract features of the intrinsic and extrinsic

coordinate frames. In a separate analysis, we trained three single-posture classifiers using data of each single posture (Pro, Sup, or Mid) and applied each of these classifiers to data of the other (i.e., Pro classifier to Sup [Pro to Sup] and Mid data [Pro to Mid],

5 Sup classifier to Pro [Sup to Pro] and Mid data [Sup to Mid], and Mid classifier to Pro [Mid to Pro] and Sup data [Mid to Sup]). Note that classification accuracies were examined for wrist-action classification. All mean classification accuracies except Pro to Mid were not significantly different from chance level (Pro to Sup: 47.8\%, $p=0.64$; Pro to Mid: $57.0 \%, p=0.03$; Sup to Pro: $48.0 \%, p=0.56$; Sup to Mid: $56.9 \%, p=0.11$; Mid to Pro: $52.2 \%, p=0.46$; Mid to Sup: $48.8 \%, p=0.84$ ), whereas within the same posture, accuracies were significantly higher than chance level (accuracy $\geq 72.0, p=$ 0.0005 for each]. This suggests that the single-posture classifiers contained a mixture of voxels with pattern information on both the intrinsic and extrinsic coordinate frames and, therefore, could not be applied to the other postures.

One important aspect of SLR is that it selects critical task-related voxels to maximize classification performance. With accuracy comparisons (Fig. 4) indicating 
that the FvE and UvD classifiers held voxel pattern information important for the intrinsic and extrinsic coordinate frames, respectively, we next examined the classifiers' weight values for the selected voxels located in the 5 focal areas of M1, PMd, PMv, pre-SMA, and SMA proper. This analysis allowed us to examine which classifications

5 were related to which brain regions. Figure 5A (left, blue gradient) depicts brain regions with univariate activation during task performance relative to the Still condition. Prominent activations were observed in the M1 hand area (Fig. 5A, left, part of the yellow-outlined area), and interestingly, the area nearly overlaps with another showing higher weight values for FvE classification (Fig. 5A, right, red regions inside 10 yellow-outlined area), suggesting that the M1 hand area was dominated by the FvE classifier.

Comparing mean focal area weight values for the FvE and UvD classifiers (Fig. 5B), we found that the M1 focal area had a significantly higher mean weight value for the FvE classifier than the UvD classifier $(p=0.02)$. Also, focal areas of PMd, PMv, and SMA proper showed significantly higher mean weight values for UvD than FvE (PMd: $p=0.001$, PMv: $p=0.007$, SMA proper: $p=0.02$ ). Pre-SMA did not show a significant difference in $\mathrm{FvE}$ and $\mathrm{UvD}$ weight values $(p=0.33)$. 


\section{Discussion}

This fMRI study examined the neural representations of the intrinsic and extrinsic coordinate frames using an MVPA method. The results demonstrate that the intrinsic and extrinsic coordinate frames for motor control are distinctively represented in M1 and PM. Prior works have examined human premotor and motor area functions during motor control (Duque et al., 2012; Eisenberg et al., 2010, 2011; Sasaki et al., 2012; Stadler et al., 2012; Strother et al., 2012; Toxopeus et al., 2011). However, the current findings, to our knowledge, are the first to demonstrate the possibility of discriminating human brain areas for intrinsic and extrinsic coordinate frames using fMRI and machine

10 learning. This study also suggests that different wrist actions (flexion and extension) and movement directions (up and down) can be classified using exclusive voxel pattern information for the intrinsic and extrinsic coordinate frames.

\subsection{Contributions to the intrinsic and extrinsic coordinate frames}

Our results revealed that the intrinsic coordinate frame was mainly associated with M1,

15 while the extrinsic coordinate frame was mainly associated with PM. This finding is reasonable, given the layered organization of the cerebral cortex and signal pathways to M1 and PM. Since M1 transmits output signals to the muscles through some layers, the 
selection of flexion or extension, which is associated with only internal body control, may be represented in M1. At the same time, the other layers of M1 receive input from PM, SMA, and the cingulate motor area, which may be the reason why direction of movement is encoded not only in PM regions but also in M1 to some degree (Eisenberg et al., 2010; Toxopeus et al., 2011). This in turn would explain why, in our analysis, M1 includes some voxels related to the extrinsic coordinate frame (Fig. 5B). It can also be argued that the mixture of intrinsic and extrinsic voxels in M1 are reflective of muscle tuning, described in Kakei and Strick (1999). However, their study used implanted electrodes, whereas the current study used surface EMG, making it difficult to dissociate voxel pattern information for muscles and joints with this experimental design.

Nonetheless, motor-related cortical regions identified in this study are consistent with existing findings.

Prior studies have shown that PMv represents not only direction of action (Eisenberg et al., 2010; Kakei et al., 2001; Toxopeus et al., 2011) but also visuomotor transformation (Lacquaniti and Caminiti, 1998; Picard and Strick, 2001). We believe our results support this view. Since the behavioral tasks in our experiment were cued with graphical arrows, visuomotor transformations from direction information in the external world to action information in the internal body would be necessary. In addition, a path 
of visuomotor transformation required for grasping was shown connecting the dorsal extrastriate cortex and PMv via the anterior intraparietal area (Rizzolatti et al., 1998). Although our experimental tasks were not finger movements, similar information may be processed for wrist flexion and extension. PMv then outputs visuomotor transformed

5 information to M1, which may also explain, the slight contribution by PMv to intrinsic coordination.

Rizzolatti and colleagues also showed that a path of visuomotor transformations required for reaching connects the parieto-occipital extrastriate area and PMd separately from the path required for grasping (Rizzolatti et al., 1998). From the significant contribution of PMd to the extrinsic coordinate frame found in our study, reaching and wrist flexion and extension may also share similar information. Other studies have also revealed that PMd is associated with motor planning and initiation (Duque et al., 2012; Hanakawa et al., 2008) and action prediction (Stadler et al., 2012). In this study, participants performed tasks according to periodic visual stimuli and had to memorize 15 the relationship between each task and respective cue. Therefore, participants might have naturally predicted and prepared for the next task, which may partly have led to PMd activation. However, this behavior would apply to all task conditions, and if PMd activation is due to motor planning, the FvE classifier should also show high weight 
values in PMd (Fig. 5B). Therefore, the PMd activity may be due to not only motor planning but also movement direction coordination. In addition, information of movement direction seems to be necessary for motor planning, and these processes may need to use extrinsic parameters. coordinate frame, but SMA proper showed significantly higher contribution to the extrinsic coordinate frame. This result is reasonable given the distinct aspects of motor control for these regions. Prior findings have shown that pre-SMA plays a role in externally cued tasks, whereas SMA proper plays a role in self-paced tasks (Deiber et al., 1991; Playford et al., 1992). Our results can be similarly interpreted considering the experimental design of the current study. Participants had to perform force exertions according to visual cues that might have involved pre-SMA function. Since the cue was commonly presented for all tasks, voxels in pre-SMA were likely selected by both FvE and UvD classifiers, resulting in no significant difference between them (Fig. 5B). In contrast, SMA proper may have been involved in task repetition during the $18 \mathrm{~s}$ task blocks, and our results suggest that task repetition required extrinsic rather than intrinsic parameters. 
Since M1 and PM include various somatotopies and sub-regions, machine learning techniques are useful for selecting voxels representing motor coordinate frames from broadly and complexly distributed areas. Among a number of MVPA methods, we utilized SLR for training classifiers and performing contribution analysis. SLR can estimate high dimensional weight values without the dimensional reduction normally needed in conventional classification methods, such as SVM. Therefore, we considered it to be the most appropriate approach to obtain a generalized model of the neurofunction. Moreover, prior findings support the validity of SLR and a feature selection method called automatic relevance determination (ARD) (Mackay, 1992) employed by SLR in neuroscientific applications. For example, a neurophysiological study using monkey neural activity data during arm movements (Tin et al., 2008) showed that more than $90 \%$ of neurons selected by the ARD method matched those selected by a standard brute-force combinational model search. Human fMRI studies involving visual tasks utilized the ARD method for feature selection and succeeded in

15 the reconstruction (Miyawaki et al., 2008) and classification (Yamashita et al., 2008) of visual stimuli, despite the cortical column structure of visual cortex. These studies suggest that SLR using the ARD method is effective in selecting neuroscientifically critical voxels, and even a single voxel may represent averaged information of the 
comprised neurons. In the current study, having confirmed strong performance and generalization for the FvE and UvD classifiers using the cross-posture analyses (Fig. 4), the selected voxels, at least, seem to be representative of a more distributed network. Thus, by comparing focal area weight values of the two classifiers, we were able to examine contributions of those areas to the intrinsic and the extrinsic coordinate frames.

\subsection{Possible dominance of movement direction information}

The current experimental design orthogonalized wrist action and movement direction representations in order to dissociate them. Our main analysis trained wrist action- and direction-specific classifiers based on multiple posture data (Pro and Sup; Fig. 4).

10 However, when trained on single posture data (Pro, Sup, or Mid only) and then applied to data from the two remaining postures, the classifiers did not show classification accuracies significantly above chance. Since it can be argued that the low accuracies of these single posture-trained classifiers were due to the inherent reduction in training data, we also tested cross- posture classifiers (FvE and UvD classifiers using Pro and

15 Sup data) trained based only on half the amount of data originally analyzed. Here, although the FvE classifier showed significantly higher accuracy than chance level (FvE classifier to Mid data: $57.2 \%, p=0.03$ ), both classifiers had significantly lower 
accuracies than the originals using Mid data (UvD classifier to Mid data: 48.3\%, $p=$ 0.002; FvE classifier to Mid data: $57.2 \%, p=0.01)$. Therefore, further investigation is needed to clarify this point.

Interestingly, however, classifiers trained by single posture tended to be direction- rather

5 than wrist action-oriented. Mean classifier accuracies were higher when the original classifiers trained on single postures acted as UvD classifiers rather than FvE classifiers. The original classifier trained with Pro data classified downward movement and flexion movement as the same class. When the original classifier was applied to Sup data, the accuracy for UvD classification tended to be higher than that for FvE classification. It is interesting that this tendency was also observed in the original analysis based on Sup data. Although further investigation is needed to formally test this tendency, it seems that classifiers trained with single posture data involve more information about movement direction than wrist action. This is probably because the information of movement direction is functionally and/or spatially dominant to that of wrist action, as the classifier is trained to maximize discriminable information by utilizing as many features (i.e., voxels) as possible. 


\section{Conclusion}

In this study, we provided the first demonstration of distributed representations of human intrinsic and extrinsic coordinate frames using MVPA. SLR analysis revealed that the most successful classifiers for intrinsic wrist action and extrinsic movement direction had ample exclusive pattern information for each classification. Analysis of weight matrices further revealed that the M1-hand area was mainly associated with the intrinsic coordinate frame, while PMd, PMv, and SMA proper were associated with the extrinsic coordinate frame. Detected activations were also consistent with existing findings, particularly for M1 and PMv. These results demonstrate the efficacy of our method in discriminating cortical representations of intrinsic and extrinsic coordinate frames and offer a step forward in non-invasive decoding of neural information.

\section{Acknowledgments}

We thank Dr. Shinji Kakei (Tokyo Metropolitan Institute of Medical Science) and Dr.

Yoichi Miyawaki (The University of Electro-Communications) for their helpful comments and Dr. David E. Vaillancourt (University of Florida) for kindly providing the HMAT template. This study is the result of "Brain Machine Interface Development" 
carried out under the Strategic Research Program for Brain Sciences by MEXT. A part of this study was supported by MEXT KAKENHI Grant Number 24500163 and CREST of the Japan Science and Technology Agency. 


\section{References}

Alaerts, K., Swinnen, S.P., Wenderoth, N., 2009. Is the human primary motor cortex activated by muscular or direction-dependent features of observed movements? , Cortex 45, 1148-1155.

Cheney, P.D., Fetz, E.E., Palmer, S.S., 1985. Patterns of Facilitation and Suppression of Antagonist Forelimb Muscles from Motor Cortex Sites in the Awake Monkey. Journal of Neurophysiology $53,805-820$.

Dafotakis, M., Sparing, R., Eickhoff, S.B., Fink, G.R., Nowak, D.A., 2008. On the role of the ventral premotor cortex and anterior intraparietal area for predictive and reactive scaling of grip force. Brain Research 1228, 73-80.

Davare, M., Andres, M., Cosnard, G., Thonnard, J.L., Olivier, E., 2006. Dissociating the role of ventral and dorsal premotor cortex in precision grasping. Journal of Neuroscience 26, 2260-2268.

Davare, M., Montague, K., Olivier, E., Rothwell, J.C., Lemon, R.N., 2009. Ventral premotor to primary motor cortical interactions during object-driven grasp in humans. Cortex 45, 1050-1057.

Deiber, M.P., Passingham, R.E., Colebatch, J.G., Friston, K.J., Nixon, P.D., Frackowiak, R.S., 1991. Cortical areas and the selection of movement: a study with positron emission tomography. Experimental Brain Research 84, 393-402.

Donoghue, J.P., Leibovic, S., Sanes, J.N., 1992. Organization of the Forelimb Area in Squirrel-Monkey Motor Cortex - Representation of Digit, Wrist, and Elbow Muscles. Experimental Brain Research 89, 1-19.

Duque, J., Labruna, L., Verset, S., Olivier, E., Ivry, R.B., 2012. Dissociating the Role of Prefrontal and Premotor Cortices in Controlling Inhibitory Mechanisms during Motor Preparation. J Neurosci $32,806-816$.

Eisenberg, M., Shmuelof, L., Vaadia, E., Zohary, E., 2010. Functional Organization of Human Motor Cortex: Directional Selectivity for Movement. J Neurosci 30, 8897-8905.

Eisenberg, M., Shmuelof, L., Vaadia, E., Zohary, E., 2011. The Representation of Visual and Motor Aspects of Reaching Movements in the Human Motor Cortex. J Neurosci 31, 12377-12384.

Evarts, E.V., 1968. Relation of Pyramidal Tract Activity to Force Exerted during Voluntary Movement. Journal of Neurophysiology 31, 14-\&.

Georgopoulos, A.P., Schwartz, A.B., Kettner, R.E., 1986. Neuronal Population Coding of Movement Direction. Science 233, 1416-1419.

Grafton, S.T., Mazziotta, J.C., Presty, S., Friston, K.J., Frackowiak, R.S., Phelps, M.E., 1992. Functional anatomy of human procedural learning determined with regional cerebral blood flow and PET. J Neurosci 12, 2542-2548.

Hanakawa, T., Dimyan, M.A., Hallett, M., 2008. Motor planning, imagery, and execution in the distributed motor network: a time-course study with functional MRI. Cereb Cortex 18, 
$2775-2788$.

Jenkins, I.H., Brooks, D.J., Nixon, P.D., Frackowiak, R.S., Passingham, R.E., 1994. Motor sequence learning: a study with positron emission tomography. J Neurosci 14, 3775-3790.

Jenkins, I.H., Jahanshahi, M., Jueptner, M., Passingham, R.E., Brooks, D.J., 2000. Self-initiated versus externally triggered movements. II. The effect of movement predictability on regional cerebral blood flow. Brain 123 ( Pt 6), 1216-1228.

Kakei, S., Hoffman, D.S., Strick, P.L., 1999. Muscle and movement representations in the primary motor cortex. Science 285, 2136-2139.

Kakei, S., Hoffman, D.S., Strick, P.L., 2001. Direction of action is represented in the ventral premotor cortex. Nature Neuroscience 4, 1020-1025.

Kamitani, Y., Tong, F., 2005. Decoding the visual and subjective contents of the human brain. Nature Neuroscience 8, 679-685.

Koike, Y., Kawato, M., 1995. Estimation of dynamic joint torques and trajectory formation from surface electromyography signals using a neural network model. Biological Cybernetics 73, 291-300.

Kriegeskorte, N., 2011. Pattern-information analysis: From stimulus decoding to computational-model testing. Neuroimage 56, 411-421.

Kurata, K., 1993. Premotor cortex of monkeys: set- and movement-related activity reflecting amplitude and direction of wrist movements. Journal of Neurophysiology 69, 187-200.

Lacquaniti, F., Caminiti, R., 1998. Visuo-motor transformations for arm reaching. European Journal of Neuroscience 10, 195-203.

Lancaster, J.L., Summerln, J.L., Rainey, L., Freitas, C.S., Fox, P.T., 1997. The Talairach Daemon, a database server for Talairach Atlas Labels. Neuroimage 5, S633.

Lancaster, J.L., Woldorff, M.G., Parsons, L.M., Liotti, M., Freitas, E.S., Rainey, L., Kochunov, P.V., Nickerson, D., Mikiten, S.A., Fox, P.T., 2000. Automated Talairach Atlas labels for functional brain mapping. Human Brain Mapping 10, 120-131.

Mackay, D.J.C., 1992. Bayesian Interpolation. Neural Computation 4, 415-447.

Maldjian, J.A., Laurienti, P.J., Burdette, J.H., 2004. Precentral gyrus discrepancy in electronic versions of the Talairach atlas. Neuroimage 21, 450-455.

Maldjian, J.A., Laurienti, P.J., Kraft, R.A., Burdette, J.H., 2003. An automated method for neuroanatomic and cytoarchitectonic atlas-based interrogation of fMRI data sets. Neuroimage 19, 1233-1239.

Matsuzaka, Y., Aizawa, H., Tanji, J., 1992. A Motor Area Rostral to the Supplementary Motor Area (Presupplementary Motor Area) in the Monkey - Neuronal-Activity during a Learned Motor Task. Journal of Neurophysiology 68, 653-662.

Mayka, M.A., Corcos, D.M., Leurgans, S.E., Vaillancourt, D.E., 2006. Three-dimensional locations and boundaries of motor and premotor cortices as defined by functional brain imaging: a meta-analysis. Neuroimage 31, 1453-1474. 
Miyawaki, Y., Uchida, H., Yamashita, O., Sato, M.A., Morito, Y., Tanabe, H.C., Sadato, N., Kamitani, Y., 2008. Visual Image Reconstruction from Human Brain Activity using a Combination of Multiscale Local Image Decoders. Neuron 60, 915-929.

Mourao-Miranda, J., Bokde, A.L.W., Born, C., Hampel, H., Stetter, M., 2005. Classifying brain states and determining the discriminating activation patterns: Support Vector Machine on functional MRI data. Neuroimage 28, 980-995.

Mur, M., Bandettini, P.A., Kriegeskorte, N., 2009. Revealing representational content with pattern-information fMRI--an introductory guide. Soc Cogn Affect Neurosci 4, 101-109.

Nichols, T.E., Holmes, A.P., 2002. Nonparametric permutation tests for functional neuroimaging: a primer with examples. Human Brain Mapping 15, 1-25.

Pereira, F., Mitchell, T., Botvinick, M., 2009. Machine learning classifiers and fMRI: a tutorial overview. Neuroimage 45, S199-209.

Pesaran, B., Nelson, M.J., Andersen, R.A., 2006. Dorsal premotor neurons encode the relative position of the hand, eye, and goal during reach planning. Neuron 51, 125-134.

15 Picard, N., Strick, P.L., 2001. Imaging the premotor areas. Current Opinion in Neurobiology 11, 663-672.

Playford, E.D., Jenkins, I.H., Passingham, R.E., Nutt, J., Frackowiak, R.S., Brooks, D.J., 1992. Impaired mesial frontal and putamen activation in Parkinson's disease: a positron emission tomography study. Annals of Neurology 32, 151-161.

Rizzolatti, G., Luppino, G., Matelli, M., 1998. The organization of the cortical motor system: new concepts. Electroencephalography and Clinical Neurophysiology 106, 283-296.

Sasaki, A.T., Kochiyama, T., Sugiura, M., Tanabe, H.C., Sadato, N., 2012. Neural networks for action representation: a functional magnetic-resonance imaging and dynamic causal modeling study. Frontiers in Human Neuroscience 6.

Scott, S.H., Kalaska, J.F., 1995. Changes in Motor Cortex Activity during Reaching Movements with Similar Hand Paths but Different Arm Postures. Journal of Neurophysiology 73, 2563-2567.

Shima, K., Aya, K., Mushiake, H., Inase, M., Aizawa, H., Tanji, J., 1991. 2 Movement-Related Foci in the Primate Cingulate Cortex Observed in Signal-Triggered and Self-Paced Forelimb Movements. Journal of Neurophysiology 65, 188-202.

Stadler, W., Ott, D.V.M., Springer, A., Schubotz, R.I., Schutz-Bosbach, S., Prinz, W., 2012. Repetitive TMS suggests a role of the human dorsal premotor cortex in action prediction. Frontiers in Human Neuroscience 6.

Stelzer, J., Chen, Y., Turner, R., 2013. Statistical inference and multiple testing correction in classification-based multi-voxel pattern analysis (MVPA): random permutations and cluster size control. Neuroimage 65, 69-82.

Stephan, K.M., Fink, G.R., Passingham, R.E., Silbersweig, D., Ceballosbaumann, A.O., Frith, C.D., Frackowiak, R.S.J., 1995. Functional-Anatomy of the Mental Representation of Upper Extremity 
Movements in Healthy-Subjects. Journal of Neurophysiology 73, 373-386.

Strother, L., Medendorp, W.P., Coros, A.M., Vilis, T., 2012. Double representation of the wrist and elbow in human motor cortex. Eur J Neurosci.

Tin, J.A., D'Souza, A., Yamamoto, K., Yoshioka, T., Hoffman, D., Kakei, S., Sergio, L., Kalaska, J., Kawato, M., Strick, P., Schaal, S., 2008. Variational Bayesian least squarest: An application to brain-machine interface data. Neural Networks 21, 1112-1131.

Toxopeus, C.M., de Jong, B.M., Valsan, G., Conway, B.A., Leenders, K.L., Maurits, N.M., 2011. Direction of Movement Is Encoded in the Human Primary Motor Cortex. PLoS One 6.

Yamashita, O., Sato, M., Yoshioka, T., Tong, F., Kamitani, Y., 2008. Sparse estimation automatically 10 selects voxels relevant for the decoding of fMRI activity patterns. Neuroimage 42, 1414-1429.

Yoshimura, N., DaSalla, C.S., Hanakawa, T., Sato, M., Koike, Y., 2012. Reconstruction of flexor and extensor muscle activities from electroencephalography cortical currents. Neuroimage 59, 1324-1337. 


\section{Figure legends}

\section{Figure 1}

(A) Schematic of participant postures and relation between movement directions and tasks according to visual instructions in three different wrist postures. The

5 MRI-compatible fixation apparatus consists of splints, Velcro tape, titanium screws, and a plastic platform. [Left panel] Pronated posture (Pro). An upward force exertion was equivalent to an extension task and cued with an up arrow (ExtUp), whereas a downward force exertion was equivalent to a flexion task and cued with a down arrow (FlexDown). [Middle panel] Supinated posture (Sup). An upward force exertion was

10 equivalent to a flexion task and cued with an up arrow (FlexUp), whereas a downward force exertion was equivalent to an extension task and cued with a down arrow (ExtDown). [Right panel] Midway posture between Pro and Sup (Mid). A left or right arrow was used for visual instruction in this posture. A leftward force exertion was equivalent to a flexion task and cued with a left arrow (FlexLeft), whereas a rightward force exertion was equivalent to an extension task and cued with a right arrow (ExtRight). (B) Block design for the fMRI experiment. Execution tasks (Flex and Ext) 
were instructed with up and down arrows (in Pro and Sup) or left and right arrows (in Mid) inside a gray box, and a no-force task (Still) was instructed with a vertical bar (in Pro and Sup) or a horizontal bar (in Mid).

\section{Figure 2}

5 Mean muscle activity levels across participants were compared among tasks. Blue bars indicate activity levels of flexor muscle during flexion tasks, and red bars indicate those of extensor muscle during extension tasks. Mean values of individual participants were normalized by the maximum values of all condition data followed by mean calculation across participants. Error bars denote standard error.

\section{$10 \quad$ Figure 3}

Data combinations used for calculating classifiers exclusively for the intrinsic (Flex vs. Ext; FvE classifier) and the extrinsic (Up vs. Down; UvD classifier) coordinate frames. For FvE classifier calculation, Flex in Pro (FlexDown) and Sup (FlexUp) were classified as Flex, and Ext in Pro (ExtUp) and Sup (ExtDown) were classified as Ext.

15 For UvD classifier calculation, Ext in Pro (ExtUp) and Flex in Sup (FlexUp) were classified as Up, and Flex in Pro (FlexDown) and Ext in Sup (ExtDown) were classified as Down. Data from Mid posture were not used for training classifiers for either 
coordinate frame but were used to test if the FvE and the UvD classifiers were actually exclusive to a coordinate frame. A classifier for FlexLeft vs. ExtRight (FLvER classifier) was calculated to confirm that data from Mid posture had enough pattern information for classification.

\section{$5 \quad$ Figure 4}

(A) Mean accuracies averaged across 10 participants for FvE classification and UvD classification using Pro and Sup data. Error bars denote standard errors. A dotted line represents chance level of 50\%. There was no significant difference between the mean accuracies of the two classifications. (B) Mean classification accuracies averaged across 10 participants using Mid data. A dotted line represents chance level of 50\%. [Left] Classification accuracy of FLvER classifier from 6-fold cross validation using Mid data. The trained classifier did not specifically discriminate information for the intrinsic (Flex vs. Ext) or the extrinsic (Left vs. Right) coordinate frames, but did discriminate mixed information (FlexLeft vs. ExtRight) since only Mid data was used for classifier training.

15 [Middle] Results using pre-calculated UvD classifiers. The UvD classifier for each participant was the mean of 6 classifiers obtained from 6 -fold cross validation using Pro and Sup data (from left bar of Fig. 4A). [Right] Results using pre-calculated FvE 
classifiers. The FvE classifier for each participant was the mean of 6 classifiers obtained from 6-fold cross validation using Pro and Sup data (from right bar of Fig. 4A). *: $p<$ $0.01, * *: p<0.005, * * *: p<0.001$ by permutation tests.

\section{Figure 5}

5 (A) [Left panel] Gradient blue areas show activation areas associated with right wrist movements revealed by an SPM group-level univariate activation analysis contrasting all tasks with baseline (uncorrected $p<0.01$ ). Colored lines represent borderlines of five HMAT-areas, M1 (yellow), PMd (orange), PMv (purple), pre-SMA (black), and SMA proper (light pink) (Mayka et al., 2006), rendered on an MNI standard brain image using MRIcroGL (http://www.mccauslandcenter.sc.edu/mricrogl/). [Right panel] Anatomical mapping of weight values of FvE (red) and UvD (green) classifiers. Voxel-wise mean weight values of all participants were calculated for FvE and UvD classifiers. (B) Anatomical mapping and comparison of mean weight values between the two classifiers in five focal areas. The focal areas were $6 \mathrm{~mm}$-radius spheres centered at

15 local maxima statistically defined with small volume correction within each of the HMAT-areas shown in (A). Upper panels illustrate univariate activation for the HMAT-areas. Middle panels show positions of the focal areas enclosed by yellow boxes 
with magnified views. The lower bar charts compare mean weights of FvE and UvD

classifiers for focal areas, with MNI coordinates. Green areas and bars represent UvD mean weight, while red areas and bars represent FvE mean weight. Statistical difference between FvE and UvD classifiers for each focal area was assessed by permutation test. $5 \quad *: p<0.05, * *: p<0.01$. Error bars denote standard error. 
A Pronated (Pro)

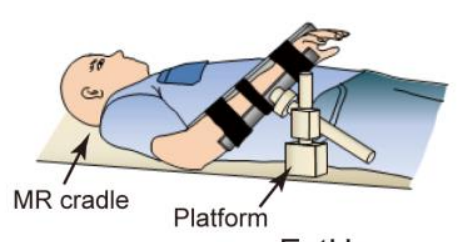

ExtUp

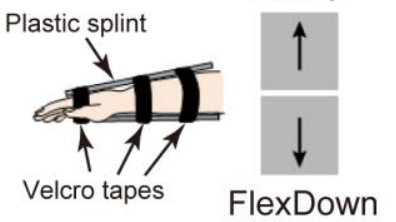

Supinated (Sup)

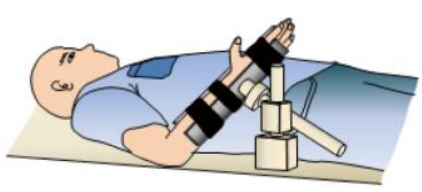

FlexUp

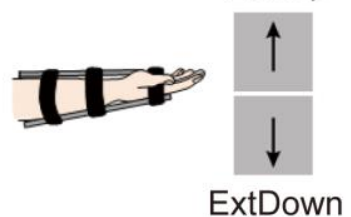

Midway (Mid)

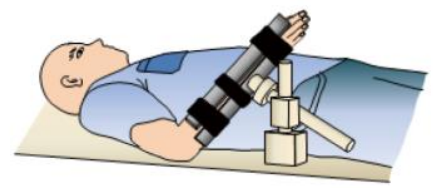

FlexLeft

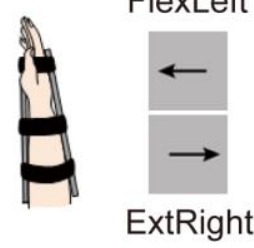

B

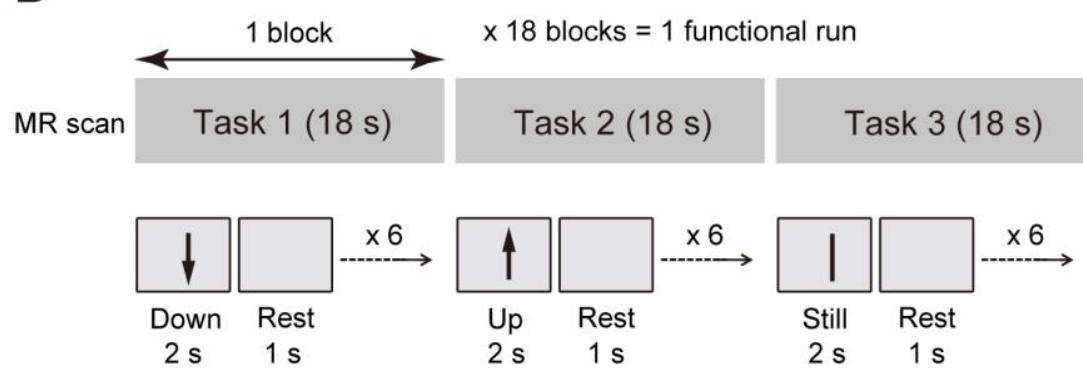

Figure 1 (Yoshimura et al.) 


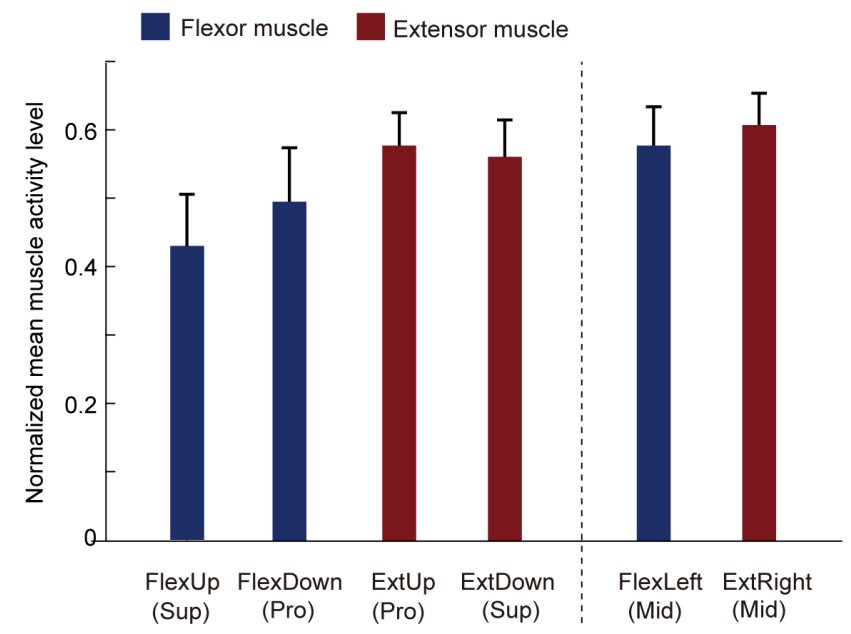

Figure 2 (Yoshimura et al.) 


\begin{tabular}{|c|c|c|}
\hline $\begin{array}{c}\text { Classifier type } \\
\text { (Coord. system) }\end{array}$ & $\begin{array}{c}\text { Data used for each class } \\
\text { in classifier calculation }\end{array}$ \\
\hline $\begin{array}{c}\text { FvE classifier } \\
\text { (Intrinsic) }\end{array}$ & Flex & \\
\hline & & \\
&
\end{tabular}

Figure 3 (Yoshimura et al.) 

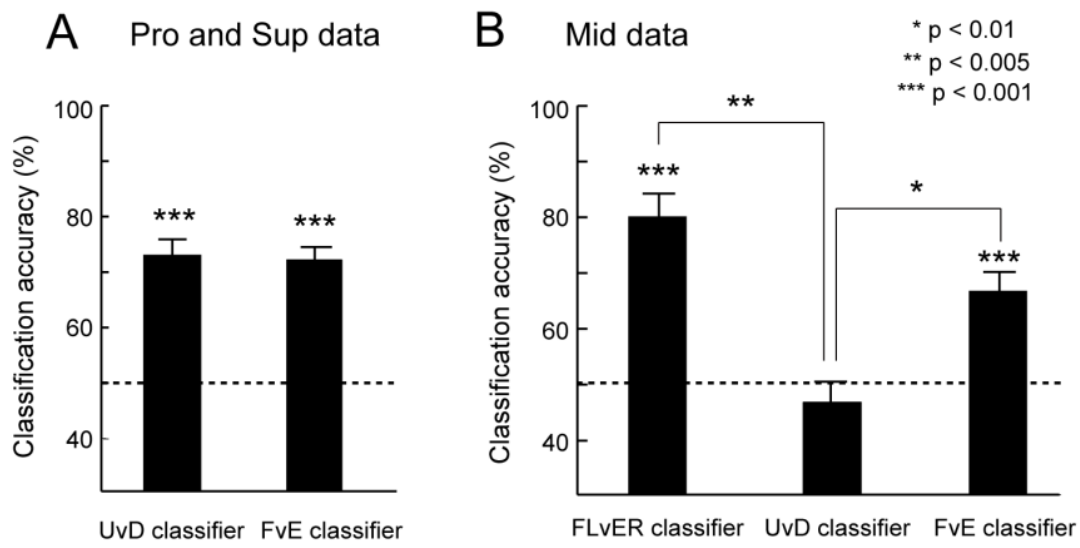

Figure 4 (Yoshimura et al.) 
A

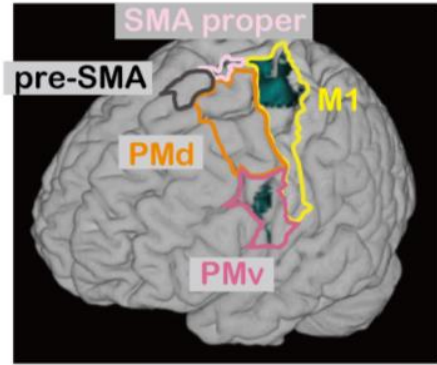

$\mathrm{T}(9)$

2.8

Uncorrected: $p<0.01$

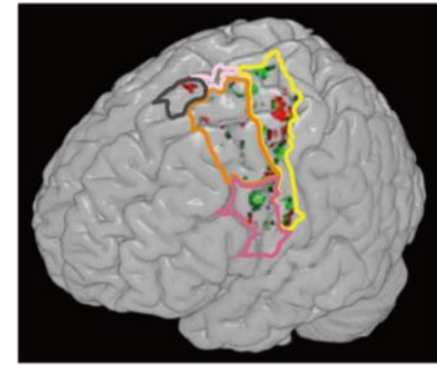

FvE weight UvD weight

B
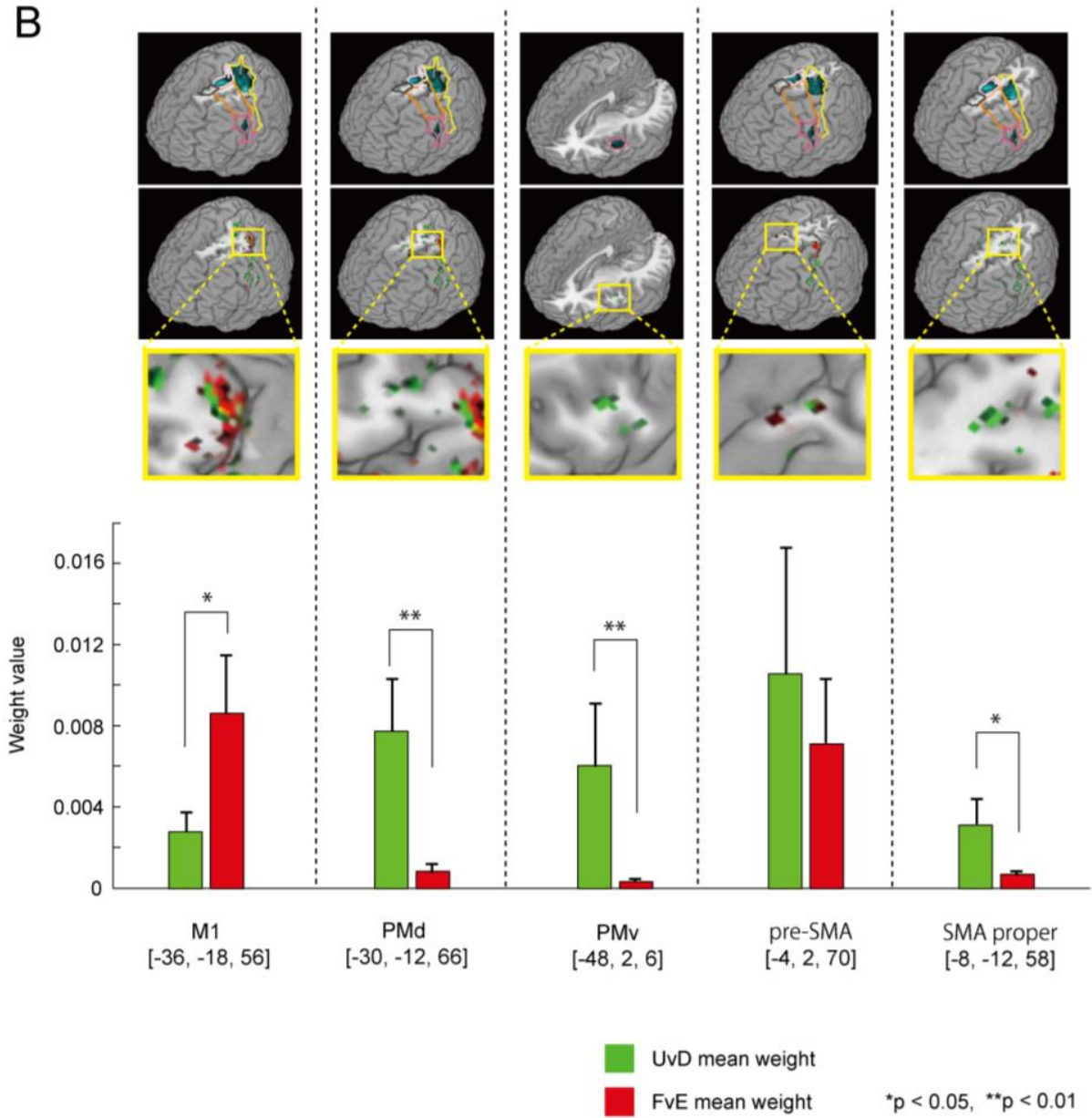

Figure 5 (Yoshimura et al.) 